Hautarzt 2018 $69: 350-351$

https://doi.org/10.1007/s00105-018-4158-x

Online publiziert: 19. April 2018

(c) Springer Medizin Verlag GmbH, ein Teil von Springer Nature 2018

CrossMark

\author{
H. F. Ständer ${ }^{1,2} \cdot$ D. Nashan ${ }^{1}$ \\ 'Hautklinik, Klinikum Dortmund gGmbH, Dortmund, Deutschland \\ ${ }^{2}$ Dermatologie Bad Bentheim, Praxis im Paulinenkrankenhaus, Bad Bentheim, Deutschland
}

\title{
Notfälle in der Dermatologie
}

Notfälle in der Dermatologie werden bei oberflächlicher Betrachtung des Faches Dermatologie allzu gerne ignoriert und übersehen. Gründe mögen sein, dass dieses Thema nicht im zentralen Fokus des primären Fachinteressierten steht und nicht der alltäglichen Arbeit zugeordnet wird.

Auf der anderen Seite hat sich die Dermatologie in den vergangenen Jahren erheblich weiterentwickelt. Die Diagnostik und Therapie der unterschiedlichen dermatologischen Erkrankungen ist komplexer geworden. Neben einer zunehmend molekularen Diagnostik ist die Dermatologie in therapeutischen Ansätzen wie dem Einsatz monoklonaler Antikörper und den zielgerichteten Therapien zum Vorreiter in der medizinischen Entwicklung geworden. Die Zahl möglicher Systemtherapien insbesondere im Bereich chronisch entzündlicher Hauterkrankungen sowie der Onkologie hat deutlich zugenommen. Damit gehen aber auch andere potenzielle Risiken durch geänderte Wechsel- und Nebenwirkungen für unsere Patienten einher, die zunehmend unseren Blick von der Haut auf eine mögliche systemische Beteiligung richten lassen.

Der erfahrene Dermatologe weiß, dass Notfälle in der Dermatologie nicht mehr selten sind und auch in der Behandlung immer anspruchsvoller werden. Damit sind nicht die allgemeinmedizinischen oder chirurgischen Notfälle gemeint, die selbstverständlich in unseren Einrichtungen ebenfalls vorkommen, sondern Notfälle, die in unserem Fachgebiet selbst entstehen, nicht zuletzt durch unsere modernen diagnostischen und therapeutischen Maßnahmen, die wir für unsere Patienten ergreifen. Die Zahl der Notfälle in den dermatologischen Abteilungen steigt kontinuierlich [1, 2].
So konnte in einem Krankenhaus in Frankreich von 2008 bis 2014 ein Anstieg an dermatologischen Notfällen um $67 \%$ beobachtet werden, wohingegen das Patientenaufkommen im gleichen Zeitraum in der allgemeinen Notfallambulanz gleich geblieben ist [1].

\section{》) Notfälle in der Dermatologie sind nicht mehr selten und werden in der Behandlung immer anspruchsvoller}

Es gibt keine einheitliche Definition, wann man von einem dermatologischen Notfall spricht. Murr et al. [3] haben als Definitionsversuch festgelegt, dass es sich bei einem dermatologischen Notfall um „eine akute oder sich verschlechternde Dermatitis in weniger als 5 Tagen handelt“. Diese Definition greift gerade in der heutigen Zeit nicht weit genug, da sie sich ausschließlich auf die Haut beschränkt, jedoch keine Schweregradeinteilung vornimmt und systemische Reaktionen nicht berücksichtigt, wie sie bei allergischen Reaktionen oder in der modernen Tumortherapie auftreten können.

Um der wachsenden Bedeutung der dermatologischen Notfälle gerecht $\mathrm{zu}$ werden, haben wir dieses Themenheft aufgelegt. Es soll das Spektrum unterschiedlicher Notfälle in der Dermatologie dargestellt werden. Allgemeine internistische oder chirurgische Notfälle sind hier bewusst nicht mit aufgenommen, da sie den Rahmen dieses Leitthemenheftes sprengen würden. Schwerpunktmäßig werden Beiträge vorgestellt, die sich mit den spezifischen Notfällen in der Allergologie, mit foudroyanten Medikamentenreaktionen, mit physikalischen 
und chemischen Notfällen der Haut, Notfällen in der Phlebologie sowie mit onkologischen Notfällen beschäftigen. Dabei sollen insbesondere die klinisch relevanten und praktischen Aspekte im Vordergrund stehen. Zur Abrundung des Themenheftes berichtet eine Assistenzärtin in der Dermatologie über eine notfallmäßige Vorstellung aus dem Nachtdienst in einer dermatologischen Klinik.

Wir hoffen, dass wir mit dieser $\mathrm{Zu}$ sammenstellung Interesse wecken, eine Aktualisierung zum Thema und eine Hilfestellung anbieten, um künftig mit diesen dermatologischen Notfällen noch professioneller umzugehen. Gerade den jüngeren Kolleginnen und Kollegen soll es eine Handlungssicherheit im Management solcher Notfälle geben.

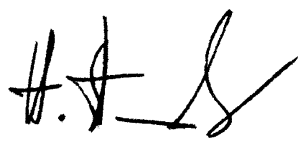

Dr. Hartmut Ständer

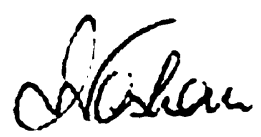

Prof. Dr. Dorothee Nashan

\section{Korrespondenzadresse}

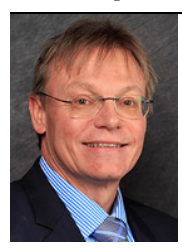

Dr. H. F. Ständer

Hautklinik, Klinikum

Dortmund gGmbH

Beurhausstr. 40, 44137 Dort-

mund, Deutschland

Hartmut.Staender@

klinikumdo.de

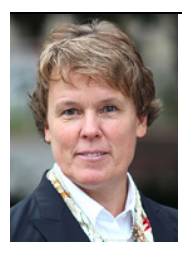

Prof. Dr. D. Nashan

Hautklinik, Klinikum

Dortmund gGmbH

Beurhausstr. 40, 44137 Dort-

mund, Deutschland

dorothee.nashan@

klinikumdo.de

Interessenkonflikt. H.F. Ständer und D. Nashan

geben an, dass kein Interessenkonflikt besteht.

\section{Literatur}

1. Isnard C, Ingen-Housz-Oro S et al (2017) Dermatological emergencies: evolution from 2008 to 2014 and perspectives. J Eur Acad Dermatol Venereol 31:274-279

2. Mirkamali A, Ingen-Housz-Oro S et al (2013) Dermatological emergencies: a comparative study of activity in 2000 and 2010. J Eur Acad Dermatol Venereol 27:916-918

3. Murr D, Bocquet $\mathrm{H}$ et al (2003) Medical activity in a emergencyoutpatient department dermatology. Ann Dermatol Venereol 130:167-170 